\title{
Sandwich-Type Zeolite Intergrowths with MFI and the Novel Extra- Large Pore IDM-1 as Ordered End-Members
}

\author{
Luis A. Villaescusa,* Jian Li, Alvaro Mayoral, Zihao Rei Gao, and Miguel A. Camblor* \\ Cite This: Chem. Mater. 2021, 33, 7869-7877 \\ Read Online
}

ABSTRACT: Stacking faults are two-dimensional planar defects frequently arising in zeolites, modifying their properties and potentially affecting their performance in catalysis and separation applications. In classical zeolite intergrowths, a topologically unique zeolite layer may often pile up after some spatial transformation (lateral translation, rotation, and/or reflection) that may occur in different amounts or directions with about similar probabilities, leading to a difficult to control disorder. Here, we present a new kind of zeolite intergrowth that requires an additional topologically distinct layer rather than a spatial transformation of a unique one. Stacking of the so-called pentasil layers produces the well-known medium pore zeolite MFI. Intercalation in

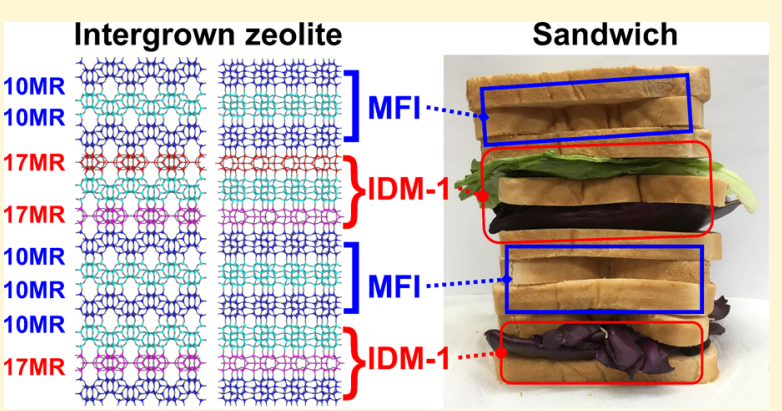
strict alternation of a topologically distinct second layer sandwiched between pentasil layers expands the structure to produce the new extra-large pore IDM-1. Stacking disorder modulates the structural expansion along the stacking direction. The disordered materials have been studied by simulation of the X-ray diffraction patterns using the program DIFFaX and by Cs-corrected highresolution electron microscopy. We show that disorder does not occur at random but in extended domains and can be controlled all the way from MFI to IDM-1 by just varying the concentration of the synthesis mixture.

\section{INTRODUCTION}

Due to their wide field of applications and their diverse and tunable properties, zeolites attract a continuous large scientific and industrial interest. ${ }^{1-4}$ While most known zeolite structures are periodically ordered in all directions, stacking faults are relatively frequent in zeolites because a zeolite layer may on occasions be assembled to the next topologically identical layer in different ways (for instance, after a rotation, a reflection and/or a translation along one or several axes by a fraction of the unit cell edge). ${ }^{5}$ When the different ways of assembly bring about no large differences in stability, the possibility of disorder along one dimension arises. Ordered stackings of the building layers give rise to ordered polymorphs, while stacking faults produce disordered, nonperiodic intergrowths. The Structure Commission of the International Zeolite Association considers 23 possible families of this kind of "classical intergrown" zeolites, although some have no real existence. ${ }^{6}$ The presence of stacking faults may affect important zeolite properties and, hence, their performance in catalysis and separation applications. For instance, in the so-called ABC-6 family, disorder may result in pore blockage, ${ }^{7}$ and it may also alter the catalytic performance of the disordered material. ${ }^{8}$ The degree of intergrowth in MFI/MEL catalysts and membranes may impact their catalytic ${ }^{9-11}$ as well as their permeation and selectivity properties, respectively. ${ }^{12} \mathrm{~A} \mathrm{Cu}$-loaded AFX/CHA intergrown material, tested as a catalyst for the selective reduction of $\mathrm{NO}_{x}$ with $\mathrm{NH}_{3}$, showed a better hydrothermal stability than the ordered Cu-AFX material, ${ }^{13}$ while COK-24, an intergrowth of Erionite and Chabazite, outperformed these ordered zeolites in the same reaction. ${ }^{14}$ Also, in ECNU-5, a disordered MWW, the degree of disorder also affects the adsorption and catalytic properties. ${ }^{15}$ Tuning the properties of zeolites for specific applications may therefore require to control their degree of intergrowth. Moreover, on occasions, it may be desirable to synthesize just one of the ordered endmembers. This would be the case of polymorph A of zeolite Beta, ${ }^{16,17}$ which is chiral and could find important applications in enantioselective catalysis and adsorption, but which has never been prepared as the ordered polymorph.

Thus, the ability to control intergrowths in zeolites is of practical as well as of fundamental importance. This is, however, generally very difficult, although there are some few examples in which intergrowths can be controlled. One strategy for such a control consists of using two or more organic structure-directing agents (OSDAs), provided that OSDAs that are specific to each end-member are known (an OSDA typically resides in the pore of the crystallized zeolite so they are used with the intention of determining the zeolite

Received: July 29, 2021

Revised: September 22, 2021

Published: September 30, 2021 
phase that crystallizes by controlling at a certain degree the size and shape of the pore space).$^{18}$ For instance, MFI/MEL and FAU/EMT intergrowths can be controlled using mixtures of tetrapropylammonium and tetrabutylamonium ${ }^{10,19}$ (or tetrapropyammonium and $\mathrm{N}, \mathrm{N}$-diethyl-3,5-dimethyl piperidinium $)^{20}$ and crown ethers $15 \mathrm{C} 5 / 18 \mathrm{C} 6$, respectively. ${ }^{20}$ Frequently, the specificity of each OSDA to one of the ordered zeolites is very dissimilar, which may limit the maximum amount of the most specific OSDA required to produce intergrowths. A very interesting case in which a combination of an OSDA and a mixture of inorganic structuredirecting agents (ISDAs) was used to control the system has been very recently reported: PST-33, the missing AABC ordered end-member of the ABC- 6 family, was synthesized by combining 5-azonia-spiro[4.4] nonane (OSDA), $\mathrm{Na}^{+}$, and $\mathrm{K}^{+}$ (ISDA). ${ }^{2}$

Other strategies may rely on changing the composition of the framework, as shown by $\mathrm{Xu}$ et al., for the -SVY/MWW intergrown zeolites, in which disorder was controlled by the $\mathrm{Si} / \mathrm{Al}$ ratio. ${ }^{22}$ Sometimes, a change of a mineralizer (fluoride substituting for hydroxide) may cause changes in the nature of the polymorphs present in the disordered material, but without further control of disorder. ${ }^{23}$ Finally, there has been one single report in which the degree of intergrowth is fully controlled from almost pure end-member to almost pure end-member using a single OSDA, no ISDA and no change in framework composition or mineralizer: the STF/SFF family. ${ }^{24}$ Intriguingly, the degree of disorder in this case is controlled by the degree of dilution of the synthesis gel and the crystallization temperature: STF is favored at high temperature and concentration, while SFF is favored at low temperature and concentration, with disordered materials crystallizing in between those limits.

Very recently, we reported a new extra-large pore silica zeolite, denoted as IDM- $1,{ }^{25}$ which can be described as an expansion of the very well-known MFI structure by intercalation of a new structural slice sandwiched between adjacent MFI layers (the so-called "pentasil" layers). The resulting material possesses two-lobed pores limited by 16- or 17 -membered rings (16MR or 17MR) depending on the occupation of a silicon site. Here, we report a new family of intergrown materials involving the classical MFI and the new IDM-1 topologies as ordered end-members. The intergrowth scheme is of a new type because it is not based on any change in the spatial orientation of the stacking layers, but rather on the presence or absence of a second type of layer, with a different thickness and topology, sandwiched between the primary pentasil layers. The degree of disorder can be controlled end-to-end from MFI to IDM-1 by simply varying the concentration of the crystallization mixture.

\section{EXPERIMENTAL SECTION}

2.1. Synthesis of the Zeolites. The OSDA, $p$-phenylenedimethylene-bis(tripropylammonium), was prepared as previously reported. ${ }^{25}$ The zeolites were crystallized from mixtures containing this OSDA in the hydroxide form, $\mathrm{SiO}_{2}$, and water using fluoride anions as mineralizing agents at near to neutral $\mathrm{pH}$. In a typical experiment, $3.47 \mathrm{~g}$ of tetraethylorthosilicate $(0.01666$ moles $)$ was added to $7.17 \mathrm{~g}$ of a solution of the OSDA hydroxide containing 1.16 $\times 10^{3}$ moles of $\mathrm{OH}^{-}$per $1000 \mathrm{~g}$ in a Teflon vessel. Hydrolysis was carried out under stirring at room temperature, keeping the vessel uncovered, which allows the evaporation of the generated ethanol and some water. When the mass of the reactant mixture was $4.83 \mathrm{~g}, 0.35 \mathrm{~g}$ of HF (48\%) was added and homogenized by hand with a spatula
[CAUTION! HF is corrosive and toxic, and it must be handled under a hood and wearing adequate personal protection equipment]. The magnet used to stir the solution was removed, and the vessel was covered before putting it in an autoclave, which was heated at $150{ }^{\circ} \mathrm{C}$. For this experiment, the calculated molar ratio of the reactant mixture was $1 \mathrm{SiO}_{2}$ : $0.25 \mathrm{OSDAF}_{2}: 8 \mathrm{H}_{2} \mathrm{O}$. After 17 days of reaction, the autoclave was taken out of the oven and cooled. The solid was obtained by filtration and washed with plenty of water. Experiments at three different temperatures $\left(135,150\right.$, and $\left.165{ }^{\circ} \mathrm{C}\right)$ and varying the $\mathrm{H}_{2} \mathrm{O} / \mathrm{SiO}_{2}$ ratios were performed, and the results are summarized in Table $\mathrm{S} 1$ and graphically in Figure 1.

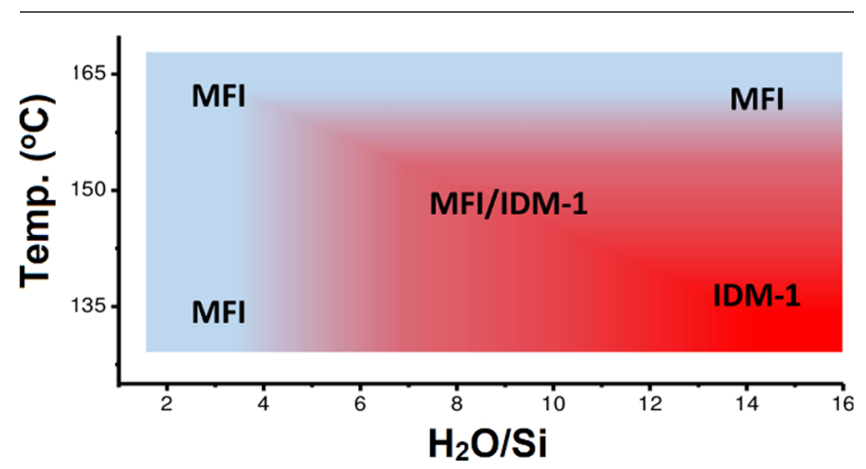

Figure 1. Graphical summary of synthesis results as the concentration and temperature vary. The areas denoted as MFI, IDM-1, and MFI/ IDM-1 refer to crystallization fields yielding essentially pure MFI, IDM-1, and intergrowths of both phases, respectively.

2.2. Characterization. Laboratory powder X-ray diffraction (PXRD) patterns were collected on a Bruker D8 Advance in Bragg-Brentano geometry with $\mathrm{Cu} \mathrm{K} \alpha$ radiation using no monochromator and a rapid position sensitive detector (lynxeye). The measuring range was 4 to $35^{\circ}$, and the total measuring time was 1 $\mathrm{h}$ per sample. Field emission scanning electron microscopy (FE-SEM) images were taken with a FEI Nova NanoSEM $230 .{ }^{29} \mathrm{Si}$ solid state nuclear magnetic resonance (NMR) spectra of the as-made zeolites were collected on a Bruker AV-400WB using long relaxation delays of $180 \mathrm{~s}$, and further details are provided elsewhere. ${ }^{26}$

2.3. Ab Initio Structure Solution by Continuous Rotation Electron Diffraction. The ab initio structure solution of the as-made IDM-1 was done similar to our previous work. ${ }^{25}$ Shortly, a droplet of suspension was transferred to a copper grid. The data were collected by a quad hybrid pixel detector (Timepix) with video mode, using the software instamatic. ${ }^{27}$ All the electron diffraction patterns were recorded under spot size 3 with exposure time $0.5 \mathrm{~s}$. Data processing was conducted using the software packages $\mathrm{XDS}^{28}$ and REDp. ${ }^{29}$ Structure solution was performed using SHELXT ${ }^{30}$ with the merged and scaled data sets, and then, the framework with $20 \mathrm{Si}$ atoms and 42 $\mathrm{O}$ atoms in the asymmetric unit was refined using Olex $2,{ }^{31}$ with atomic scattering factors for electrons.

2.4. Rietveld Refinement. Synchrotron PXRD patterns were collected at the Material Science Powder Diffraction beamline BL04MSPD in the Spanish synchrotron facility, ALBA, in Debye-Scherrer geometry with a wavelength $\lambda=0.61866795 \AA$. The Rietveld refinement of the as-made IDM- 1 was performed using data up to a dspacing of $0.9044 \AA$ with Topas 6.1. ${ }^{32}$ The model from continuous rotation electron diffraction (cRED) data was used as the initial model. Before starting the refinement, the background of the diffraction pattern was removed. In order to retrieve the residual electron density in the channel system, the scale factor and the framework of IDM-1 were refined using the high angle data. The residual electron density in the channel system can be obtained by one cycle of refinement with all parameters fixed using the whole $2 \theta$ range. Then, the OSDA molecule was introduced into the structural model as a rigid body to perform the global optimization with a simulated annealing algorithm. During the global optimization, all the parameters were fixed, except the location and orientation of the 
OSDA. A reliable starting position of the ODSA in the channel system could be quickly obtained. Next, all the parameters were opened to refine one by one, such as peak shape, unit cell, instrument parameters, position of framework atoms, and torsion angles in the OSDA molecule. It should be noted here that soft restraints were placed on the $\mathrm{Si}-\mathrm{O}$ bond distances $(1.61 \AA)$, the $\mathrm{Si}-\mathrm{O}-\mathrm{Si}$ bond angles $\left(145^{\circ}\right)$, and $\mathrm{O}-\mathrm{Si}-\mathrm{O}$ bond angles $\left(109^{\circ}\right)$. These restraints were gradually reduced. Finally, the unit cell dimensions, background, and peak profile were further refined with the original synchrotron data. The Rietveld refinement of as-made MFI was performed in a similar way.

2.5. Scanning Transmission Electron Microscopy (STEM). The samples were prepared by deeply crushing the powder for several minutes using a mortar and pestle. The materials were then dispersed in methanol and ultrasonicated. Few drops of the suspension were placed onto holey carbon copper microgrids. Transmission electron microscopy analyses were carried out in a FEI-TITAN XFEG operated at $300 \mathrm{kV}$. The microscope was operated in scanning transmission mode, and the aberrations were minimized using a CEOS spherical aberration corrector, assuring a spatial resolution of 0.8 A. Images were collected using an annular dark field (ADF) detector. For image simulations, a supercell of $69.973 \times 39.982 \times$ $280.445 \AA^{3}$ was created using the already reported CIF file data of IDM- $1 .{ }^{25}$ The quantitative scanning transmission electron microscopy (QSTEM) simulation software was used, based on the multislice method. ${ }^{33}$ The data presented here correspond to a crystal thickness of $12-20 \mathrm{~nm}$. The parameters used were $C_{\mathrm{s}}=0.001 \mathrm{~mm}, U_{\text {acc }}=300$ $\mathrm{kV}$, collection angle 50-200 mrad, and convergence semiangle 17 mrad.

2.6. DIFFaX Simulations. Intergrowths were studied by comparison of the experimental synchrotron $(\lambda=0.619274 \AA$, collected at MSPD in ALBA) or $\mathrm{Cu}$ K $\alpha$ PXRD patterns with the ones simulated using DIFFaX, a fortran program that calculates the diffraction intensities of crystals containing stacking faults. ${ }^{34,35}$ Several different models were tested, and the details of two of them (a fourlayer random model and a six-layer clustered model) are provided in the Supporting Information.

\section{RESULTS AND DISCUSSION}

3.1. Zeolite Synthesis. Depending on the temperature and water to silica ratio, some products of the crystallization of silica in the presence of $p$-phenylenedimethylene-bis(tripropylammonium) and fluoride could be clearly identified by PXRD as IDM-1 or MFI, while others had features that suggested an intergrowth between those phases rather than a phase mixture (Figure 1; also see synthesis results in Table S1). At high temperature and also at lower temperature, but in concentrated conditions, materials identifiable as MFI were obtained, while lower temperatures and more diluted conditions afforded the new extra-large pore zeolite IDM- $1 .{ }^{25}$ The crystallization of MFI in this system is not totally unexpected because our OSDA is formally close to a dimer of the most typical OSDA for this zeolite (tetrapropylammonium) with a rigid $p$-xylylene linker.

The PXRD patterns of a representative series of calcined zeolites obtained by varying the $\mathrm{H}_{2} \mathrm{O} / \mathrm{SiO}_{2}$ ratio from 3 to 15 at $150{ }^{\circ} \mathrm{C}$ are shown in Figure 2 (see Figure $\mathrm{S} 1$ for the as-made patterns). Contrary to the patterns of the MFI and IDM- 1 endmembers, the patterns of the in-between materials are characterized by the presence of some peaks with varying intensity and width. For instance, two reflections at around 5.0 and $7.05^{\circ}$ are absent in MFI, broad in the intermediate phases, and sharp and intense in IDM-1 [reflections (020) and (011), respectively]. Also, a $10.04^{\circ}$ peak that is the sharp reflection (040) in IDM-1 and is absent in MFI appears as a low intensity broad reflection that moves up from slightly smaller angles,

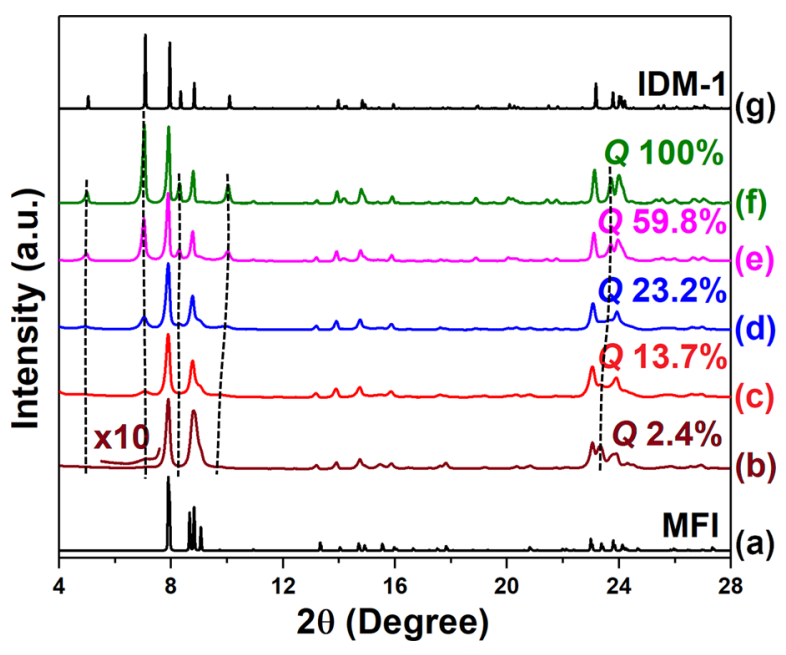

Figure 2. $\mathrm{Cu} \mathrm{K} \alpha$ PXRD patterns of two simulated end-members and five calcined materials obtained at increasing degrees of dilution of the synthesis mixture at $150{ }^{\circ} \mathrm{C}$ : (a) simulated MFI end-members and samples obtained from (b) $\mathrm{H}_{2} \mathrm{O} / \mathrm{SiO}_{2}=3$ for 14 days, (c) $\mathrm{H}_{2} \mathrm{O} / \mathrm{SiO}_{2}$ $=6.5$ for 20 days, (d) $\mathrm{H}_{2} \mathrm{O} / \mathrm{SiO}_{2}=8$ for 17 days, (e) $\mathrm{H}_{2} \mathrm{O} / \mathrm{SiO}_{2}=9$ for 17 days, (f) $\mathrm{H}_{2} \mathrm{O} / \mathrm{SiO}_{2}=15$ for 38 days and (g) simulated IDM-1 end-members. The $Q$ values (see text and Supporting Information) of each sample are marked. The dotted lines mark the position of reflections that vary in position, broadness, or intensity along the series (see text).

while becoming sharper and more intense as the degree of dilution increases. The area around $23-25^{\circ}$ is also characterized by broad and overlapped reflections in the intermediate materials, with some few peaks also shifting position: the (50-1) and (501) reflections of MFI, appearing around 23.3 and $23.4^{\circ}$, merge and broaden, while slightly shifting to higher angles in the intermediate materials, finally becoming the reflection (091) of IDM-1 at around $23.8^{\circ}$. All this points to the existence of an intergrowth series between the MFI and IDM-1 end-members, with the degree of intergrowth being controlled by the degree of dilution of the synthesis mixture at low and intermediate temperatures (135 and $150{ }^{\circ} \mathrm{C}$ ) but not at high temperature $\left(165^{\circ} \mathrm{C}\right.$, see Figure S2). Both MFI and IDM-1 (Figure 3a,b) contain topologically identical pentasil layers (Figure 3d), making the intergrowth of zeolites possible (Figure 3c). As shown in the figure, the main difference between different materials is the absence of the new expanding "idm" layer in MFI (Figure 3e), its strict presence sandwiched between pentasil layers in IDM-1, or its occasional existence in the intergrown intermediate materials. The idm layer is not only topologically different from the pentasil layer but also significantly thinner (around 25\% thinner).

The fact that most PXRD reflections do not appreciably change position along the intergrown series (Figure 2) suggests an intergrowth in domains sufficiently large to diffract coherently. Interestingly, the synthesis results do not meaningfully change with increasing the crystallization time, that is, once a fully crystalline material is obtained, its PXRD pattern hardly changes over time (Figure S3). A similar behavior was observed in the STF/SFF family. ${ }^{24}$ The lack or small extension of Ostwald ripening might suggest that the stabilities of all the phases involved are not largely different.

A convenient way to compare the relative proportion of IDM-1 in these materials is by the ratio of peak areas of the reflections around 7 and $7.9^{\circ} 2 \theta$ of the calcined materials 

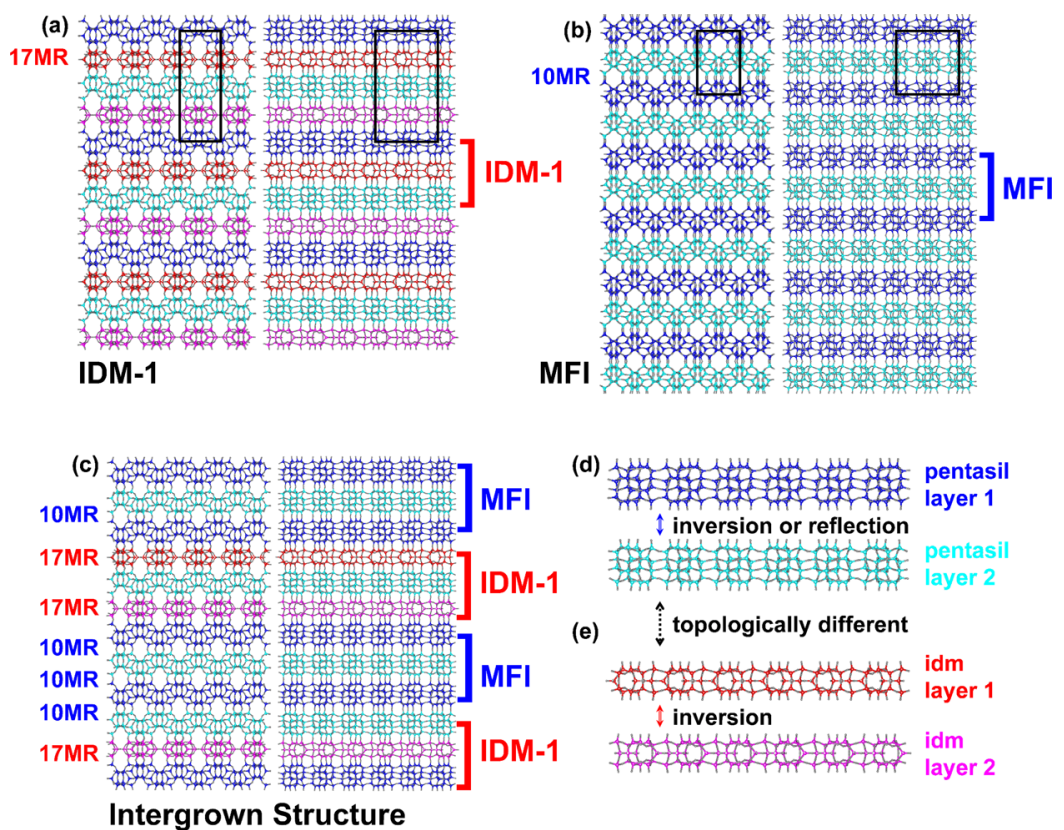

Intergrown Structure

Figure 3. Structure description of the sandwich-type intergrowth. Zeolite framework along [100] and [001] directions of (a) IDM-1 end-member, (b) MFI end-member, and (c) MFI/IDM-1 intergrown sample. (d, e) Topologically different pentasil layers and idm layers, respectively.

[corresponding to the (011) and (101) reflections of IDM-1]. For calcined IDM-1, the quotient between both areas is close to one, while that for MFI is zero, as there is no reflection at $7^{\circ}$. Values in percentage $(Q)$ are given in Figure 2 for a set of samples prepared at $150{ }^{\circ} \mathrm{C}$.

3.2. Structure Elucidation of the Ordered EndMembers. The structure of the as-made IDM-1 ordered end-member has been determined by ab initio methods using 3D electron crystallography data collected by the cRED method. The most notable finding is that the as-made IDM-1 does not have a significant concentration of $Q^{2}$ sites ( $\mathrm{Si}$ connected to two $\mathrm{Si}$ ), but only $Q^{3}$ and $Q^{4}$ (connected to three and four $\mathrm{Si}$, respectively), which agrees with the ${ }^{29} \mathrm{Si}$ magicangle spinning (MAS) NMR spectrum (Figure S4); this is in sharp contrast with the structure of the calcined material, which contains $Q^{2}, Q^{3}$, and $Q^{4}{ }^{25}$ This implies that the calcination brings about a significant reorganization of Si sites. Consequently, all the extra-large pores in the as-made IDM-1 are $17 \mathrm{MR}$ pores. The structure of IDM-1 is reminiscent of the structure of zeolite SSZ-61 (*-SSO), especially in the as-made form. ${ }^{36}$ Both are extra-large pore zeolites with double-lobed pores that contain $Q^{3}$ sites along the pore wall.

The OSDA cations have been located by Rietveld refinement in both IDM-1 and MFI using synchrotron PXRD data, rigid bodies, and simulated annealing techniques. In IDM-1, there is a cation in each lobe of the two-lobed $17 \mathrm{MR}$ pore with the propylammonium moieties sitting close to the intersection with the straight medium pore channels (Figure S5). The situation in MFI is similar, with the propylammonium moieties close to the channel intersections and the benzene ring in the sinusoidal channel (Figure S6). The most striking difference is that the benzene ring lies almost flat in the $a b$ plane in IDM-1, while in MFI, it is significantly tilted (around $50^{\circ}$ ) (Figure S7). Crystallographic details and the refinement of the zeolites are summarized in the Supporting Information (Tables S2-S8 and Figures S8-S10).

3.3. DIFFaX Simulations. The intergrowth scheme in this family of disordered materials is graphically represented in
Figure 3. The intermediate disordered materials have been characterized using PXRD data and high-resolution STEM (HR-STEM) techniques. Simulation of their PXRD patterns was performed with DIFFaX. ${ }^{34,35}$ A totally random four-layer model involving two pentasil layers (related by a mirror plane and yielding MFI if directly connected; Figure S11) and two of the new idm layers (related by an inversion center and yielding IDM-1 if they strictly alternate with the pentasils) reproduced well both end-members but failed to account for the observed changes in the PXRD patterns of the intermediate materials (Table S9 and Figures S12-S14). Most notably, the (011) reflection of IDM-1, appearing around $7^{\circ}$ in all the experimental materials, arises as a shoulder of the $7.9^{\circ}$ reflection of MFI, the (-101) plus (011) reflections, and moves to lower values, while narrowing and increasing in intensity as the proportion of IDM-1 increases. This failure of the four-layer random model and the observation that most reflections are invariant in position along the experimental disordered series prompted us to consider the possibility of domain clustering, in which transition probabilities between IDM-1 and MFI layers, rather than being totally random, would be subjected to a "memory effect" favoring extended coherent domains of MFI-like and IDM-1-like stackings. In fact, there is a significant difference in size in the plane normal to the stacking of layers between the pentasils present in MFI and IDM-1 (+0.4 and $-2.2 \%$ in different directions) which, in our opinion, would produce a small misfit that could be a reasonable cause for this memory effect: a pentasil (or its associated idm) layer of a given size/kind would tend to be followed by a pentasil of the same size/kind, giving rise to extended domains that would reduce the occasions in which misfits occur. To simulate clustered domains, we built a sixlayer model, fully described in the Supporting Information, where two additional pentasil layers would represent extended MFI domains. In this model, there could be three different probabilities to govern the layer transitions, but we fixed the $\alpha$ probability of an IDM-1 pentasil to be followed by an idm layer to almost unity (0.99) to produce IDM-1 domains. Then, 
the probability $\beta$ would govern the transition from idm layers to MFI pentasils. Finally, $\gamma$ will govern the probability that an MFI domain prevails (Table S10).

We thus simulated a set of patterns varying $\beta$ and $\gamma$ to cover a whole range of existence probability of each phase, while maintaining $\beta$ as high as possible (this was found to be a requirement for a good fit and confirms that domains are generally large; see Figure S15). The resulting patterns are shown in Figure 4. The trends observed for simulated and

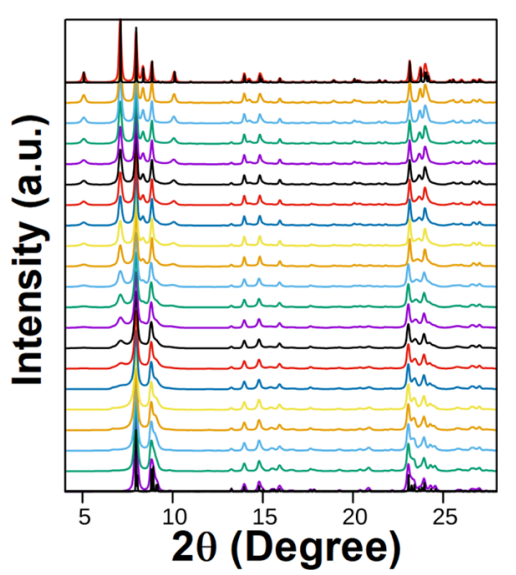

Figure 4. $\mathrm{Cu} \mathrm{K} \alpha$ PXRD DIFFaX simulations (color traces) of clustered MFI/IDM-1 intergrowth models. The IDM-1 existence probability is (from bottom) 1 , from 5 to 95 in $5 \%$ increments, and $98 \%$. The $\beta$ and $\gamma$ probabilities used are given in Table S10. The patterns of calcined MFI and IDM-1, as simulated by VESTA, ${ }^{37}$ are shown close to the 1 and $98 \%$ probabilities as black lines.

experimental patterns as the percentage of IDM-1 increases are in pretty good agreement, supporting the idea of clustered domains. This demonstrates that DIFFaX can handle very well the PXRD simulation of this new type of nonclassical intergrowths.

We observed, however, a small disagreement between the experimental and simulated patterns for the most intermediate samples: the area around $23.3-23.8^{\circ}$, where peaks move along the disordered series, is rather flat in those samples and might in fact contain several overlapped reflections. Such a small disagreement may arise, in our opinion, from the unlikeliness of a very uniform sample composed of crystals all with the same degree of intergrowth and with the same size of domains. A mixture of crystals is more likely and, thus, a combination of several simulations could agree better with the experimental pattern. To check this idea and, at the same time, challenge our models, we tried to simulate the high-resolution synchrotron PXRD data of the intermediate sample with $Q=23 \%$. We found that a 0.7:0.3 mixture of the simulations with $(\beta, \gamma)$ pairs of $(0.9,0.85)$ and $(0.62,0.9)$ reproduced quite reasonably the main features of the high-resolution pattern, as shown in Figure 5, proving our point. $C_{\mathrm{s}}$-corrected STEM-ADF analysis of the same sample supports this heterogeneity of crystals having different proportions/extensions of domains (see below).

The effect of the intergrowths in the textural properties of the zeolites is clearly observed in their $\mathrm{N}_{2}$ adsorption isotherms: a step with hysteresis at a relative pressure around 0.2 , characteristic of MFI, ${ }^{38}$ becomes less and less noticeable and finally vanishes as the proportion of IDM-1 increases (Figure S16).

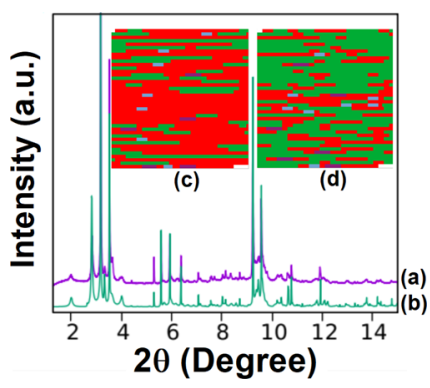

Figure 5. High-resolution synchrotron $(\lambda=0.619274 \AA)$ PXRD patterns of the sample with $Q=23 \%$ (purple, a) and a 0.7:0.3 combination of DIFFaX clustered simulations with $(\beta, \gamma)$ pairs of $(0.9,0.85)$ and $(0.62,0.9)$ (green, $b)$. The colorful $c$ and $d$ areas represent the sequence of layers generated by the computer when using the Random 1022 instruction in the DIFFaX files used to generate these PXRD patterns. ${ }^{35}(\beta, \gamma)$ pair values are $(0.62,0.9)(\mathrm{c})$ and $(0.9,0.85)(d)$. Red and green backgrounds denote IDM-1 and MFI domains, respectively, while pentasil diads or triads are distinctly highlighted in blue and purple, respectively, to note their existence, though they are scarce. The figures demonstrate the generation of a wide distribution of domains that, while containing mainly extended domains, also contain very small ones.

3.4. HR-STEM Study. Next, we investigated by electron microscopy both end-members and the intermediate sample with $Q=23 \%$ (Figure 6). Due to the similarity between MFI and IDM-1 in the direction normal to the stacking of layers (which is the direction of the straight channels in both zeolite structures and the $b$ direction in IDM-1), no differences among the three samples were observed along the [010] projection by high-resolution imaging. We shall use here the axis setting of IDM-1 when referring to crystallographic axis. Figure 6a shows the spherical aberration $C_{\mathrm{s}}$-corrected STEM-ADF micrograph of the edge of a typical IDM-1 crystal. The yellow arrows in the bottom inset point to two consecutive pentasil chains, which are related by an inversion center to finally yield the pentasil layer. By recording data on crystallites tilted $90^{\circ}$, that is, along the $c$-axis, distinct differences with respect to the MFI type became apparent (Figure 6b). A closer look is depicted in Figure 6c, which clearly evidenced the IDM-1 framework, formed by a succession of pentasil and idm layers. For direct interpretation, the simulated framework, based on the previously reported model, is shown in an inset. ${ }^{25}$

Data on the intergrown sample is presented in Figure 7. Figure $7 \mathrm{a}$ shows the image along the $b$-axis, with no visual differences with either IDM-1 or MFI structures. Figure $7 \mathrm{~b}$ depicts another crystal, which was tilted $90^{\circ}$ along the $c$-axis. In this case, three regions can be identified, two of them corresponding to pure IDM-1 forming the largest domains, while a smaller one is associated to MFI. The Fourier diffractogram, shown in the inset of Figure $7 b$, displays diffuse streaks along the $b^{*}$-axis, as a result of the existence of stacking faults in the crystal, rather than the well-defined spots observed for IDM-1. A closer observation of the intergrowth is shown in Figure $7 c$, where the existence of a small three-pentasil MFI domain (marked by yellow dashed arrows) enclosed by two large regions of the IDM-1 framework can be unambiguously visualized. The schematic model of both structures is superimposed on top of the image, showing a perfect match between the models and the experimental data. Additional lower magnification micrographs of different crystallites, recorded along the $[201]$ projection, still allowing the 

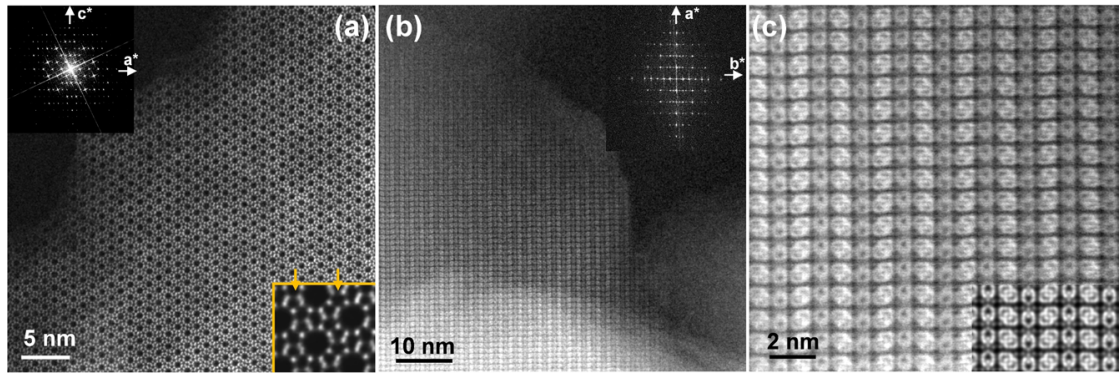

Figure 6. $C_{\mathrm{s}}$-corrected STEM-ADF images of IDM-1: (a) along $b$-axis (an enlarged image of the framework is presented in the bottom right inset, framed by yellow lines. The yellow arrows point at the pentasil chains), (b) high-resolution image along the $c$-axis, (c) magnified image obtained from (b) with the simulated micrograph superimposed, bottom right. The insets in (a) and (b) show the corresponding Fourier diffractogram.

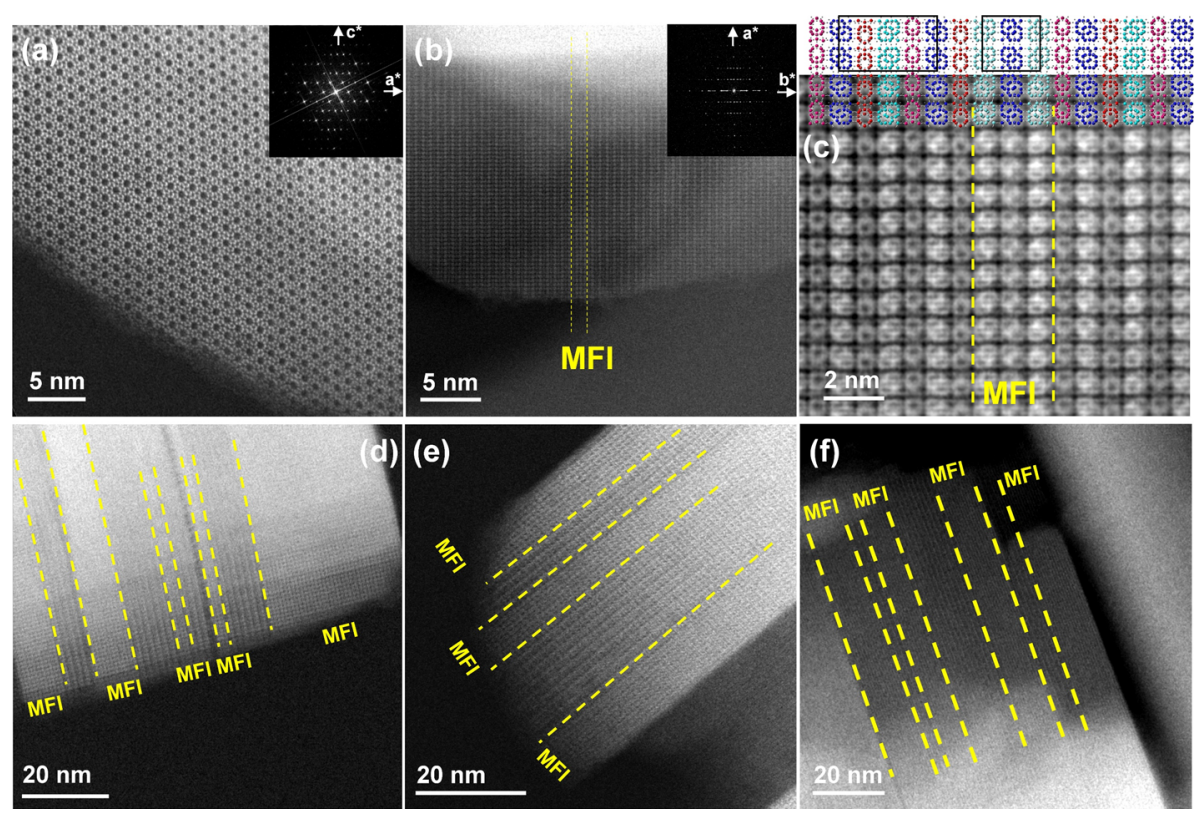

Figure 7. $C_{\mathrm{s}}$-corrected STEM-ADF images of the intergrown material with $Q=23 \%$ : (a) atomic-resolution observation along the $b$-axis, $(\mathrm{b})$ data recorded along the $c$-axis, where two dashed yellow lines, indicated as MFI, mark the intergrowth, (c) atomic resolution data of the intergrowth (dashed yellow lines), where the structural models (only Si atoms) of IDM-1 and MFI with their unit cells marked are superimposed on top of the image, $(\mathrm{d}-\mathrm{f}$ ) different crystallites sitting along the [201] orientation (only MFI domains have been marked, while the unmarked correspond to IDM-1). The insets in (a) and (b) show the corresponding Fourier diffractogram.

framework visualization, are shown in Figure $7 \mathrm{~d}-\mathrm{f}$ and illustrate a significant heterogeneity of the crystallites in this sample and a tendency to large domains, in line with the DIFFaX conclusions. The estimated percentage of MFI in the intergrowths shown in Figure $7 c-f$, are 2, 62, 52, and 65\%, respectively. The existence of isolated pentasil triads, as observed in Figure 7c, is compatible with our extended domain model. This is shown in the colorful areas of Figure 5, a graphical representation of two explicit sequences of random layers generated by DIFFaX using the same $(\beta, \gamma)$ pairs used to simulate the PXRD of this sample, where in addition to extended MFI or IDM-1 domains, triads and even diads of pentasil layers are observed, although in very small proportions ( $<1 \%$ for both triads and diads).

Finally, the MFI end-member was imaged with atomic resolution along the $b$-axis, clearly identifying all rings (Figure S17), without significant differences with IDM-1 along this projection, as expected. Due to the very thin plate morphology of the particles, we did not succeed in acquiring information in directions perpendicular to the stacking of layers. We note that the morphology and orientation of these MFI materials imply a very short diffusion path along the straight $10 \mathrm{MR}$ pores, which may be of importance in catalysis and membrane applications. This is further supported by FE-SEM (Figure S18), showing that the MFI-like sample consists of very thin small plates with round borders, a morphology very atypical for MFI. The plates themselves, which are usually no larger than $4 \times 5 \times 0.3 \mu \mathrm{m}$, appear to be composed of even smaller domains. Larger plates are found in the intergrown and IDM-1 samples, but in all cases the plates are normal to the direction of the stacking of different layers and therefore expose the straight MFI and IDM-1 pores.

3.5. Further Discussion. Due to its outstanding shape selectivity properties, MFI, discovered around half a century ago, is one of the most studied zeolites. It is known to intergrow "classically" (i.e., without intervention of additional topologically distinct layers) with zeolite MEL. ${ }^{9-12,39}$ Our work reveals, to our surprise, that new phenomena about MFI can still be discovered, like the possibility of nonclassical stacking disorder shown here. The new type of intergrowth in the MFI/IDM-1 family can be finely controlled all the way from one ordered end-member to the other one by a single 
synthetic parameter, the concentration of the synthesis mixture, provided the crystallization temperature is not too high. This is similar to the case of the STF/SFF family mentioned above (although, in that case, varying both temperature and concentration was needed to control the intergrowths). ${ }^{24}$ The effect of temperature simply suggests that MFI and STF are more stable than IDM-1 and SFF, respectively. The effect of concentration is more difficult to rationalize. This is an important parameter that on occasions determines the zeolite that crystalizes. ${ }^{40,41}$ We have pointed out in previous works a possible link between concentration and disorder: for example, the degree of disorder in ISV (not due to stacking faults but, possibly, to local defects) clearly increased as the concentration of the synthesis gel decreased. ${ }^{42}$ Another trend that we observed is that decreasing the concentration of the synthesis mixture frequently leads to intergrown disordered materials before producing dense phases at even higher dilution, a feature that we interpreted as a loss of specificity in the structure direction as the concentration decreases. ${ }^{43}$ However, in both cases considered here, specificity is fully recovered at some point of increased dilution to yield pure IDM-1 or SFF, respectively, leaving our observations puzzling. The fact that the additional layer needed to build IDM-1 from pentasils shows a low degree of polymerization and has an abundance of $\mathrm{Si}-\mathrm{OH}$ groups seems to broadly agree, at least intuitively, with that phase being favored at higher dilutions. Also, a dual specificity might be related, possibly, to a change in conformation of the OSDA or to a change in the OSDA-OSDA interactions, leading to a different structural direction effect. The group of S. B. Hong at POSTECH has provided abundant examples, in which the specificity of a flexible OSDA can be tuned by modifying the inorganic components of the reaction mixture and their concentrations, to the point of establishing the conformational control of highly flexible OSDAs as an alternative strategy for the discovery of new zeolites. ${ }^{44-49}$ However, the conformation of our OSDA in IDM-1 and MFI is not radically different. We believe that further studies on these two systems might give clues for a better understanding of the relationship between crystallization parameters and intergrowths.

\section{CONCLUSIONS}

This work demonstrates a new kind of intergrowth phenomenon in zeolites that clearly differs from classical zeolite intergrowths. Instead of a spatial transformation of a given zeolite layer, the intergrowth observed here is related to the presence or absence of idm, a second, topologically distinct, type of layer sandwiched between the well-known pentasil layers. Both intervening layers have also a significantly different thickness (idm is $25 \%$ thinner than pentasils). Despite the new nature of this intergrowths, the DIFFaX program is able to adequately simulate the PXRD pattern of the disordered materials. These simulations strongly suggest a not totally random disorder but a memory effect, resulting in extended domains. We rationalize this effect as a way of minimizing stress associated to a small size misfit between layers belonging to IDM-1 and MFI domains. The HR-STEM results confirm these conclusions. The degree of intergrowth may be controlled all the way from MFI to IDM-1 by just varying the water to silica ratio in the synthesis mixture.

\section{ASSOCIATED CONTENT}

\section{Supporting Information}

The Supporting Information is available free of charge at https://pubs.acs.org/doi/10.1021/acs.chemmater.1c02631.

Synthesis details, PXRD patterns, ${ }^{29} \mathrm{Si}$ MAS NMR spectra, structural details and OSDA location, cRED data information, Rietveld refinement plots, DIFFaX simulation details (model and patterns), $\mathrm{N}_{2}$ adsorption isotherms, $C_{s}$-STEM images, and FE-SEM images (PDF)

Crystallography information of as-made IDM-1 [CCDC 2092768] (CIF)

Crystallography information MFI [CCDC 2092769] (CIF)

\section{AUTHOR INFORMATION}

\section{Corresponding Authors}

Luis A. Villaescusa - Instituto Interuniversitario de Investigación de Reconocimiento Molecular y Desarrollo Tecnológico (IDM), Universitat de Valencia-Universitat Politènica de Vatencia, Valencia 46022, Spain; CIBER de Bioingeniería Biomateriales y Nanomedicina (CIBER-BBN), Valencia 46022, Spain; Departamento de Química, Universitat Politecnica de Vatencia, Valencia 46022, Spain; Email: lvillaes@qim.upv.es

Miguel A. Camblor - Instituto de Ciencia de Materiales de Madrid, Consejo Superior de Investigaciones Cientificas (ICMM-CSIC), Madrid 28049, Spain; ○orcid.org/00000001-9591-3432; Email: macamblor@icmm.csic.es

\section{Authors}

Jian Li - Berzelii Center EXSELENT on Porous Materials, Department of Materials and Environmental Chemistry, Stockholm University, Stockholm 10691, Sweden; Present Address: Department of Fiber and Polymer Technology School of Engineering Sciences in Chemistry, Biotechnology and Health KTH Royal Institute of Technology, Tekninkringen 56-58, Stockholm SE-100 44, Sweden (J.L.); 이이. orcid.org/0000-0003-2221-2285

Alvaro Mayoral - Instituto de Nanociencia y Materiales de Aragon (INMA-CSIC), Universidad de Zaragoza, Zaragoza 50009, Spain; Laboratorio de Microscopias Avanzadas (LMA-Universidad de Zaragoza), Center for High-resolution Electron Microscopy (ChEM), School of Physical Science and Technology, ShanghaiTech University, Pudong, Shanghai 201210, China; Oorcid.org/0000-0002-5229-2717

Zihao Rei Gao - Instituto de Ciencia de Materiales de Madrid, Consejo Superior de Investigaciones Científicas (ICMM-CSIC), Madrid 28049, Spain

Complete contact information is available at: https://pubs.acs.org/10.1021/acs.chemmater.1c02631

\section{Author Contributions}

L.A.V. and M.A.C. designed the experiments. L.A.V. performed the synthesis work. J.L. solved the structures and made the Rietveld refinement. A.M. performed the $C_{\mathrm{s}}$-STEM. M.A.C. carried out the DIFFaX simulations. L.A.V., Z.R.G., and J.L. carried out the characterization. M.A.C. prepared the manuscript. All the authors discussed the scientific results and commented on and revised the manuscript and supplementary information materials. 


\section{Funding}

The authors thank funding from the Spanish Ministry of Science, Innovation and Universities, under Projects MAT2015-71117-R (MINECO/FEDER, UE), PID2019105479RB-I00 and RTI2018-101599-B-C22 (MCUI/AEI/ FEDER, UE), the Swedish Research Council (VR), and the Knut and Alice Wallenberg Foundation (KAW). L.A.V. also thanks the Generalitat Valenciana (project PROMETEO/ 2018/024). A.M. acknowledges the Spanish Ministry of Science (RYC2018-024561-I) and the Regional government of Aragon (DGA E13_20R), the National Natural Science Foundation of China (NFSC-21850410448 and NSFC21835002), and the Centre for High-resolution Electron Microscopy (C $\hbar E M)$, supported by SPST of ShanghaiTech University under contract No. EM02161943.

\section{Notes}

The authors declare the following competing financial interest(s): One of the authors, L.A.V., has filed a patent on the new zeolite IDM-1 and the disordered family of zeolites reportedin this article.

\section{ACKNOWLEDGMENTS}

Synchrotron experiments were performed at beamline BL04 (MSPD) at the ALBA Synchrotron with the collaboration of ALBA staff, and a special thanks is due to A. Manjón for support in collecting the data and for helpful comments and suggestions. M.A.C. warmly thanks C. Baerlocher for helpful comments.

\section{REFERENCES}

(1) Weckhuysen, B. M.; Yu, J. Recent Advances in Zeolite Chemistry and Catalysis. Chem. Soc. Rev. 2015, 44, 7022-7024.

(2) Rangnekar, N.; Mittal, N.; Elyassi, B.; Caro, J.; Tsapatsis, M. Zeolite membranes - a review and comparison with MOFs. Chem. Soc. Rev. 2015, 44, 7128-7154.

(3) Mintova, S.; Jaber, M.; Valtchev, V. Nanosized microporous crystals: emerging applications. Chem. Soc. Rev. 2015, 44, 7207-7233.

(4) Chen, L.-H.; Sun, M.-H.; Wang, Z.; Yang, W.; Xie, Z.; Su, B.-L. Hierarchically Structured Zeolites: From Design to Application. Chem. Rev. 2020, 120, 11194-11294.

(5) Willhammar, T.; Zou, X. Stacking disorders in zeolites and openframeworks-structure elucidation and analysis by electron crystallography and X-ray diffraction. Z. Kristallogr. Cryst. Mater. 2013, 228, $11-27$.

(6) Intergrowth Families, Database of Zeolite Structure, Structure Commission of the International Zeolite Association. https://europe.izastructure.org/IZA-SC/intergrowth_table.html. (accessed 2021-0617).

(7) Millward, G. R.; Ramdas, S.; Thomas, J. M. On the direct imaging of offretite, cancrinite, chabazite and other related ABC-6 zeolites and their intergrowths. Proc. R. Soc. Lond. A 1985, 399, 5771.

(8) Chen, N. Y.; Schlenker, J. L.; Garwood, W. E.; Kokotailo, G. T. TMA-offretite. Relationship between Structural and Catalytic Properties. J. Catal. 1984, 86, 24-31.

(9) Conte, M.; Xu, B.; Davies, T. E.; Bartley, J. K.; Carley, A. F.; Taylor, S. H.; Khalid, K.; Hutchings, G. J. Enhanced selectivity to propene in the methanol to hydrocarbons reaction by use of ZSM-5/ 11 intergrowth zeolite. Microporous Mesoporous Mater. 2012, 164, 207-213.

(10) Francesconi, M. S.; López, Z. E.; Uzcátegui, D.; González, G.; Hernández, J. C.; Uzcátegui, A.; Loaiza, A.; Imbert, F. E. MFI/MEL intergrowth and its effect on n-decane cracking. Catal. Today 2005, 107-108, 809-815.
(11) Kokotailo, G. T. Catalytic Conversion with Crystalline Zeolite Product Constituting ZSM-5/ZSM-11 Intermediates. US Patent 4,289,607, 1981.

(12) Kumar, P.; Kim, D. W.; Rangnekar, N.; Xu, H.; Fetisov, E. O.; Ghosh, S.; Zhang, H.; Xiao, Q.; Shete, M.; Siepmann, J. I.; Dumitrica, T.; McCool, B.; Tsapatsis, M.; Mkhoyan, K. A. One-dimensional intergrowths in two-dimensional zeolite nanosheets and their effect on ultra-selective transport. Nat. Mater. 2020, 19, 443-449.

(13) Naraki, Y.; Ariga, K.; Nakamura, K.; Okushita, K.; Sano, T. ZTS-1 and ZTS-2: Novel intergrowth zeolites with AFX/CHA structure. Microporous Mesoporous Mater. 2017, 254, 160-169.

(14) Sree, S. P.; Verheyen, E.; De Prins, M.; Van der Donck, T.; van Tendeloo, L.; Schuetze, F.; Martens, J. A. Synthesis of a New Zeolite, Intergrowth of Erionite and Chabazite. ACS Mater. Lett. 2021, 3, $658-662$

(15) Xu, L.; Ji, X.; Jiang, J.-G.; Han, L.; Che, S.; Wu, P. Intergrown Zeolite MWW Polymorphs Prepared by the Rapid DissolutionRecrystallization Route. Chem. Mater. 2015, 27, 7852-7860.

(16) Treacy, M. M. J.; Newsam, J. M. Two new three-dimensional twelve-ring zeolite frameworks of which zeolite beta is a disordered intergrowth. Nature 1988, 332, 249-251.

(17) Newsam, J. M.; Treacy, M. M. J.; Koetsier, W. T.; de Gruyter, C. B. Structural characterization of zeolite beta. Proc. R. Soc. Lond. A 1988, 420, 375-405.

(18) Gómez-Hortigüela, L. Insights into the Chemistry of Organic Structure-Directing Agents in the Synthesis of Zeolitic Materials; Springer, 2018.

(19) Millward, G. R.; Ramdas, S.; Thomas, J. M.; Barlow, M. T. Evidence for Semi-regularly Ordered Sequences of Mirror and Inversion Symmetry Planes in ZSM-5/ZSM-11 Shape-selective Zeolitic Catalysts. J. Chem. Soc., Faraday Trans. 2 1983, 79, 10751082.

(20) John, N. S.; Stevens, S. M.; Terasaki, O.; Anderson, M. W. Evolution of Surface Morphology with Introduction of Stacking Faults in Zeolites. Chem. - Eur. J. 2010, 16, 2220-2230.

(21) Lee, H.; Choi, W.; Choi, H. J.; Hong, S. B. PST-33: A FourLayer ABC-6 Zeolite with the Stacking Sequence AABC. ACS Mater. Lett 2020, 2, 981-985.

(22) Xu, L.; Ma, T.; Shen, Y.; Wang, Y.; Han, L.; Chaikittisilp, W.; Yokoi, T.; Sun, J.; Wakihara, T.; Okubo, T. Rational Manipulation of Stacking Arrangements in Three-Dimensional Zeolites Built from Two-Dimensional Zeolitic Nanosheets. Angew. Chem., Int. Ed. 2020, 59, 19934-19939.

(23) Camblor, M. A.; Barrett, P. A.; Díaz-Cabañas, M. J.; Villaescusa, L. A.; Puche, M.; Boix, T.; Pérez, E.; Koller, H. High silica zeolites with three-dimensional systems of large pore channels. Microporous Mesoporous Mater. 2001, 48, 11-22.

(24) Villaescusa, L. A.; Zhou, W.; Morris, R. E.; Barrett, P. A. Synthesis, characterization and control of faulting in STF/SFF topologies, a new family of intergrowth zeolites. J. Mater. Chem. 2004, 14, 1982-1987.

(25) Villaescusa, L. A.; Li, J.; Gao, Z.; Sun, J.; Camblor, M. A. IDM1 , a zeolite with intersecting medium and extra-large pores built as an expansion of zeolite MFI. Angew. Chem., Int. Ed. 2020, 59, 1128311286.

(26) Rojas, A.; Martínez-Morales, E.; Zicovich-Wilson, C. M.; Camblor, M. A. Zeolite Synthesis in Fluoride Media: Structure Direction toward ITW by Small Methylimidazolium Cations. J. Am. Chem. Soc. 2012, 134, 2255-2263.

(27) Smeets, S.; Wang, B. stefsmeets/instamatic 1.6.0, Zenodo. doi: DOI: 10.5281/zenodo.4072976. (accessed 2020-06-02).

(28) Kabsch, W. Integration, scaling, space-group assignment and post-refinement. Acta Crystallogr. D Biol. Crystallogr. 2010, 66, 133144

(29) Wan, W.; Sun, J.; Su, J.; Hovmöller, S.; Zou, X. Threedimensional rotation electron diffraction: software RED for automated data collection and data processing. J. Appl. Crystallogr. 2013, 46, 1863-1873. 
(30) Sheldrick, G. M. SHELXT-Integrated space-group and crystalstructure determination. Acta. Crystallogr., A Found. Adv. 2015, 71, 38.

(31) Dolomanov, O. V.; Bourhis, L. J.; Gildea, R. J.; Howard, J. A. K.; Puschmann, H. OLEX2: A complete structure solution, refinement and analysis program. J. Appl. Crystallogr. 2009, 42, 339-341.

(32) Coelho, A. A. TOPAS and TOPAS-Academic: an optimization program integrating computer algebra and crystallographic objects written in C++. J. Appl. Crystallogr. 2018, 51, 210-218.

(33) Koch, C. T. Determination of Core Structure Periodicity and Point Defect Density along Dislocations; Ph.D. thesis, Arizona State University: Arizona, U.S., 2002.

(34) Treacy, M. M. J. DIFFaX v. 1812 Manual, 2005.

(35) Treacy, M. M. J.; Newsam, J. M.; Deem, M. W. A General Recursion Method for Calculating Diffracted Intensities from Crystals Containing Planar Faults. Proc. R. Soc. Lond. A 1991, 433, 499-520.

(36) Smeets, S.; Xie, D.; Baerlocher, C.; McCusker, L. B.; Wan, W.; Zou, X.; Zones, S. I. High-Silica Zeolite SSZ-61 with DumbbellShaped Extra-Large-Pore Channels. Angew. Chem., Int. Ed. 2014, 53, 10398-10402.

(37) Momma, K.; Izumi, F. VESTA 3 for three-dimensional visualization of crystal, volumetric and morphology data. J. Appl. Crystallogr. 2011, 44, 1272-1276.

(38) Müller, U.; Reichert, H.; Robens, E.; Unger, K. K.; Grillet, Y.; Rouquerol, F.; Rouquerol, J.; Pan, D.; Mersmann, A. High-resolution sorption studies of argon and nitrogen on large crystals of microporous zeolite ZSM-5. Fresenius' Z. Anal. Chem. 1989, 333, 433-436.

(39) Perego, G.; Cesari, M.; Allegra, G. Ordered and Disordered Structures in Borosilicates with a Pentasil-Type Framework. J. Appl. Crystallogr. 1984, 17, 403-410.

(40) Camblor, M. A.; Villaescusa, L. A.; Díaz-Cabañas, M. J. Synthesis of all-silica and high-silica molecular sieves in fluoride media. Top. Catal. 1999, 9, 59-76.

(41) Zones, S. I.; Darton, R. J.; Morris, R.; Hwang, S. J. Studies on the Role of Fluoride Ion vs Reaction Concentration in Zeolite Synthesis. J. Phys. Chem. B 2005, 109, 652-661.

(42) Villaescusa, L. A.; Díaz, I.; Barrett, P. A.; Nair, S.; LLorisCormano, J. M.; Martínez-Mañez, R.; Tsapatsis, M.; Liu, Z.; Terasaki, O.; Camblor, M. A. Pure Silica Large Pore Zeolite ITQ-7: Synthetic Strategies, Structure-Directing Effects, and Control and Nature of Structural Disorder. Chem. Mater. 2007, 19, 1601-1612.

(43) Villaescusa, L. A. Factores Directores de Estructura en la Sintesis de Zeolitas en Medio Fluoruro. Nuevas Zeolitas; Ph.D. thesis, Universidad Politécnica de Valencia: Valencia, Spain, 1999.

(44) Lee, S.-H.; Shin, C.-H.; Yang, D.-K.; Ahn, S.-D.; Nam, I.-S.; Hong, S. B. Reinvestigation into the synthesis of zeolites using diquaternary alkylammonium ions $\left(\mathrm{CH}_{3}\right)_{3} \mathrm{~N}^{+}\left(\mathrm{CH}_{2}\right)_{n} \mathrm{~N}^{+}\left(\mathrm{CH}_{3}\right)_{3}$ with $\mathrm{n}=3-10$ as structure-directing agents. Microporous Mesoporous Mater. 2004, 68, 97-104.

(45) Lee, S.-H.; Lee, D.-K.; Shin, C.-H.; Park, Y.-K.; Wright, P. A.; Lee, W. M.; Hong, S. B. Synthesis, characterization, and catalytic properties of zeolites IM-5 and NU-88. J. Catal. 2003, 215, 151-170.

(46) Shin, J.; Hong, S. B. N,N,N,N',N',N'-hexamethylpentanediammonium-MWW layered precursor: A reaction intermediate in the synthesis of zeolites TNU-9 and EU-1. Microporous Mesoporous Mater. 2009, 124, 227-231.

(47) Hong, S. B.; Min, H.-K.; Shin, C.-H.; Cox, P. A.; Warrender, S. J.; Wright, P. A. Synthesis, Crystal Structure, Characterization, and Catalytic Properties of TNU-9. J. Am. Chem. Soc. 2007, 129, 1087010885 .

(48) Lee, S.-H.; Shin, C.-H.; Choi, G. J.; Park, T.-J.; Nam, I.-S.; Han, B.; Hong, S. B. Zeolite synthesis in the presence of flexible diquaternary alkylammonium ions $\left(\mathrm{C}_{2} \mathrm{H}_{5}\right)_{3} \mathrm{~N}^{+}\left(\mathrm{CH}_{2}\right)_{n} \mathrm{~N}^{+}\left(\mathrm{C}_{2} \mathrm{H}_{5}\right)^{3}$ with $\mathrm{n}=3-10$ as structure-directing agents. Microporous Mesoporous Mater. 2003, 60, 237-249.

(49) Hong, S. B. Use of Flexible Diquaternary Structure-directing Agents in Zeolite Synthesis: Discovery of Zeolites TNU-9 and TNU-
10 and Their Catalytic Properties. Catal. Surv. Asia 2008, 12, 131144. 